\title{
NONLINEAR $p$-LAPLACIAN PROBLEMS ON UNBOUNDED DOMAINS
}

\author{
LAO SEN YU
}

(Communicated by Barbara L. Keyfitz)

ABstract. We consider the $p$-Laplacian problem

$$
\begin{gathered}
-\operatorname{div}\left(a(x)|\nabla u|^{p-2} \nabla u\right)+b(x)|u|^{p-2} u=f(x, u), \\
x \in \Omega,\left.u\right|_{\partial \Omega}=0, \lim _{|x| \rightarrow \infty} u=0,
\end{gathered}
$$

where $1<p<n, \Omega\left(\subset R^{n}\right)$ is an exterior domain. Under certain conditions, we show the existence of solutions for this problem via critical point theory.

\section{INTRODUCTION}

This paper is devoted to the study of the $p$-Laplacian problems

$$
\begin{gathered}
l u=f(x, u) \quad \text { in } \Omega, \\
\left.u\right|_{\partial \Omega}=0, \quad \lim _{|x| \rightarrow \infty} u=0,
\end{gathered}
$$

where $\Omega$ is a smooth exterior domain in $R^{n}$ (i.e., $\Omega$ is the complement of a bounded domain with $C^{1, \delta}$ boundary, $\left.0<\delta<1\right), l u=-\operatorname{div}\left(a(x)|\nabla u|^{p-2} \nabla u\right)$ $+b(x)|u|^{p-2} u, 1<p<n, 0<a_{0} \leq a(x) \in L^{\infty}(\Omega) \cap C^{\delta}(\bar{\Omega}), 0 \leq b(x) \in$ $L^{\infty}(\Omega) \cap C(\Omega)$. The objective is to obtain sufficient conditions on $f$ for $(*)$ to have positive solutions in the following three prototype cases:

$$
f(x, u)= \begin{cases}g(x) u^{\alpha}, & p-1<\alpha<p^{*}-1 ; \\ h(x) u^{\beta}, & 0 \leq \beta<p-1 ; \\ g(x) u^{\alpha}+h(x) u^{\beta}, & 0 \leq \beta<p-1<\alpha<p^{*}-1,\end{cases}
$$

where $p^{*}=n p /(n-p)$ is the Sobolev critical exponent. When $p=2,(*)$ is the usual second order elliptic problem, and (1), (2), and (3) correspond to the superlinear, sublinear, and mixed sub-superlinear cases respectively.

Several studies have appeared. For the case of bounded domains, we mention the works of Azorero and Alonso [2], Egnell [5], Guedda and Veron [6], and references therein. As to unbounded domains, we recall the results of BidautVeron [4], Li and Yan [8], and Ni and Serrin [11]. Ni and Serrin [11] studied the radial case $\operatorname{div}\left(|\nabla u|^{p-2} u\right)+u^{\alpha}=0$ in $R^{n}$ and showed that this equation

Received by the editors July 24, 1990 and, in revised form, January 22, 1991.

1991 Mathematics Subject Classification. Primary 35J60; Secondary 35J70.

Key words and phrases. $p$-Laplacian problems, decaying solutions.

Research supported by NSERC Canada. 
admits no positive radial ground state solutions if $0<\alpha<p^{*}-1$ and, conversely, it does admit one if $\alpha \geq p^{*}-1$. Li and Yan [8] considered the eigenvalue problem $\operatorname{div}\left(|\nabla u|^{p-2} \nabla u\right)+f(x, u, \lambda)=0, x \in R^{n}$, with $f(x, u, \lambda)=$ $g(x, u)-\lambda|u|^{p-2} u$ or $\lambda\left(g(x, u)-|u|^{p-2} u\right)$, assuming $\lim _{t \rightarrow 0} g(x, t) / t^{p-1}=0$, and obtained a decaying solution for $\lambda=\lambda_{0}$. Bidaut-Veron [4] studied the behaviour of solutions of $(*)$.

No existence theory seems to have been found to date for nonradially symmetric $p$-Laplacian problems of type $(*)$ in the cases (1), (2), and (3). As the problem $(*)$ has a variational structure, we naturally apply critical point theory to it. We first set up some weighted spaces in which the solutions are to be sought and for which the norm $\|u\|_{l}=\left(\int_{\Omega} a(x)|\nabla u|^{p}+b(x)|u|^{p}\right)^{1 / p}$ induced by the operator $l$ is an equivalent norm. Then we employ Mountain Pass arguments to obtain the existence of solutions. To prove the decay of the solutions, we make use of the estimates of Serrin [13].

\section{Problem (*) in Case (1)}

In this section, we consider problem $(*)$ in the case (1); that is, $f$ is of the form $g(x) t^{\alpha}$ with $p-1<\alpha<p^{*}-1$. We choose the function space $E$ as the completion of $C_{0}^{\infty}(\Omega)$ under the norm $\|u\|=\left(\int_{\Omega}|\nabla u|^{p}+\omega|u|^{p}\right)^{1 / p}$, where $\omega(x)=\max \left\{b(x), 1 /(1+|x|)^{p}\right\}$. From the definition, it is clear that $E \sim$ $W_{0}^{1, p}(\Omega)$ if $b(x) \geq b_{0}>0$. Moreover, $E$ has the following three important properties:

(a) $E$ can be embedded into $W_{\text {loc }}^{1, p}(\Omega)$;

(b) Sobolev Inequality: $\|u\|_{p^{*}} \leq A\|\nabla u\|_{p}$ for all $u \in E$, where $p^{*}=$ $n p /(n-p)$ and

$$
A=\frac{n-1}{n v_{n}^{1 / n}} \frac{\Gamma(n / p-1)}{\Gamma(n / p)},
$$

the Sobolev embedding constant (see $\left[10\right.$, p. 56]), and $v_{n}=\operatorname{vol}\left(B_{1}(0)\right)$;

(c) The norm $\|u\|_{l}=\left(\int_{\Omega} a|\nabla u|^{p}+b|u|^{p}\right)^{1 / p}$ induced by the operator $l$ is an equivalent norm on $E$. ity

Indeed, (a) and (b) are obvious, while (c) follows from a Hardy-type inequal-

$$
\int_{\Omega} \frac{1}{(1+|x|)^{p}}|u|^{p} \leq C \int_{\Omega}|\nabla u|^{p}
$$

for all $\varphi \in C_{0}^{\infty}(\Omega)$. This inequality can be obtained by applying the Divergence Theorem and Hölder inequality to the integral

$$
\begin{aligned}
\int_{\Omega} \frac{1}{(1+|x|)^{p}}|u|^{p} & =-\frac{1}{n} \int x \cdot \nabla\left(\frac{1}{(1+|x|)^{p}}|u|^{p}\right) \\
& \leq \frac{p}{n} \int_{\Omega}\left(\frac{1}{(1+|x|)^{p-1}}|u|^{p-1}\right)(|\nabla u|)+\frac{p}{n} \int_{\Omega} \frac{1}{(1+|x|)^{p}}|u|^{p} .
\end{aligned}
$$

We assume that $f$ satisfies the following conditions:

(i) $f \in C^{0}\left(\Omega \times R^{+}\right), f(x, t)>0$ in $\Omega_{0} \times(0, \infty)$ for some nonempty open $\Omega_{0} \subseteq \Omega$;

(ii) $|f(x, t)| \leq g(x)|t|^{\alpha}, p-1<\alpha<p^{*}-1,(0 \not \equiv) g \in L^{\infty} \cap L^{p_{0}}(\Omega)$ where $p_{0}=\frac{n p}{n p-(\alpha+1)(n-p)}$; 
(iii) there exists $\mu>p$ such that $\mu F(x, t) \leq t f(x, t),(x, t) \in \Omega \times R^{+}$, where $F(x, t)=\int_{0}^{t} f(x, s) d s$.

Now we define two functionals $K(u)$ and $J(u)$ on $E$,

$$
K(u)=\int_{\Omega} F(x, u) d x, \quad J(u)=\frac{1}{p}\|u\|_{l}^{p}-K(u) .
$$

$K(u)$ and $J(u)$ are well defined by assumption (ii) and Sobolev's Inequality. The functional $K(u)$ has the following basic properties.

Lemma 1. Under (i), (ii),

(a) $K(u)$ is weakly lower semicontinuous and differentiable on $E$ with $K^{\prime}(u)(\varphi)=\int_{\Omega} f(u, x) \varphi d x$ for $\varphi \in E$.

(b) $K^{\prime}(u)$ is a continuous and compact map from $E$ to $E^{*}$, the dual of $E$.

Proof. We select $\Omega_{k}=\left\{x \in \Omega|| x \mid \leq r_{k}\right\}$, where $r_{k}$ could be a fixed number or a sequence.

(a) Let $u_{j} \rightarrow u$ weakly in $E$. Observe that

$$
\begin{aligned}
\left|K\left(u_{j}\right)-K(u)\right| \leq \int_{\Omega_{k}} \mid & F\left(x, u_{j}\right)-F(x, u) \mid \\
& +C\|g\|_{L^{p_{0}\left(\Omega \backslash \Omega_{k}\right)}}\left(\left\|u_{j}\right\|_{l}^{\alpha+1}+\|u\|_{l}^{\alpha+1}\right) .
\end{aligned}
$$

Since $\left\{u_{j}\right\}$ is bounded in $E,\left\{\left.u_{j}\right|_{\Omega_{k}}\right\}$ is bounded in $W^{1, p}\left(\Omega_{k}\right)$ for fixed $k$. It follows from the compact embedding $W^{1, p}\left(\Omega_{k}\right) \hookrightarrow L^{q}\left(\Omega_{k}\right)$ for $1 \leq q<p^{*}$ (see, e.g., [1, Theorem 6.2]), that there exists a subsequence of $\left\{u_{j}\right\}$ that converges to $u$ in $L^{q}\left(\Omega_{k}\right)$, whence $u_{j} \rightarrow u$ in $L^{q}\left(\Omega_{k}\right)$. Further, $\int_{\Omega_{1}} F\left(x, u_{k}\right) \rightarrow$ $\int_{\Omega_{1}} F(x, u)$, since $|F(x, t)| \leq \frac{1}{\alpha+1} g(x)|t|^{\alpha+1}$ and $1<\alpha+1<p^{*}$. Therefore, $K\left(u_{j}\right) \rightarrow K(u)$, since $(5)$ and $g \in L^{p_{0}}(\Omega)$.

For differentiability of $K$, we show that given any $\varepsilon>0$, there exists a $\delta=\delta(\varepsilon, u)>0$ such that

$$
\left|\int_{\Omega} F(x, u+\varphi)-\int_{\Omega} F(x, u)-\int_{\Omega} f(x, u) \varphi\right|<\varepsilon\|\varphi\|_{l}
$$

for all $\varphi \in E$ with $\|\varphi\|_{l} \leq \delta$. Observe that $g \in L^{p_{0}}(\Omega)$ and

$$
\begin{aligned}
& \left|\int_{\Omega \backslash \Omega_{k}} F(x, u+\varphi)-F(x, u)-f(x, u) \varphi\right| \\
& \quad \leq \int_{\Omega \backslash \Omega_{k}} g\left\{(|u|+|\varphi|)^{\alpha}|\varphi|+|u|^{\alpha}|\varphi|\right\} \\
& \leq C\|g\|_{L^{p_{0}\left(\Omega \backslash \Omega_{k}\right)}}\left(\|u\|_{l}^{\alpha}+\|\varphi\|_{l}^{\alpha}\right)\|\varphi\|_{l}<\frac{\varepsilon}{2}\|\varphi\|_{l}
\end{aligned}
$$

for sufficiently large $r_{k}$ and $\|\varphi\|_{l} \leq 1$. To estimate the integral on the bounded domain $\Omega_{k}$ and obtain

$$
\left|\int_{\Omega_{k}} F(x, u+\varphi)-F(x, u)-f(x, u) \varphi\right|<\frac{\varepsilon}{2}\|\varphi\|_{l},
$$

we need only follow the arguments in Proposition B10 of [12]. 
(b) The continuity of $K^{\prime}(u)$ follows from the estimate

$$
\begin{aligned}
\left\|K^{\prime}\left(u_{j}\right)-K^{\prime}(u)\right\|_{E^{*}} \leq C & \left\{\left\|f\left(\cdot, u_{j}\right)-f(\cdot, u)\right\|_{L^{n p /(n+p)}\left(\Omega_{k}\right)}\right. \\
& \left.+\|g\|_{L^{p_{0}}\left(\Omega \backslash \Omega_{k}\right)}\left(\left\|u_{j}\right\|_{l}^{\alpha}+\|u\|_{l}^{\alpha}\right)\right\},
\end{aligned}
$$

$1<\alpha n p /(n+p)<p^{*}$, and arguments similar to the one above. To show the compactness, we employ the diagonal method. Let $\left\{u_{j}\right\}$ be a bounded sequence in $E$, and let $r_{k} \rightarrow \infty$ such that $\|g\|_{L^{p_{0}\left(\Omega \backslash \Omega_{k}\right)}}<\frac{1}{k}$. For each $k$, the compactness of the embedding $W^{1, p}\left(\Omega_{k}\right) \hookrightarrow L^{q}\left(\Omega_{k}\right)\left(1 \leq q<p^{*}\right)$ and the boundedness of $\left\{u_{j}\right\}$ in $W^{1, p}\left(\Omega_{k}\right)$ imply that $\left\{u_{j}\right\}$ has a Cauchy subsequence $\left\{u_{j k}\right\}$ in $L^{q}\left(\Omega_{k}\right)$. Then $\left\{K^{\prime}\left(u_{j j}\right)\right\}$ is a Cauchy sequence in $E^{*}$. Indeed, for given $\varepsilon>0$, choose $k$ such that $\|g\|_{\left.L^{p_{0}}(\Omega) \Omega_{k}\right)}<\varepsilon$. On the other hand, $\left\{u_{j j}\right\} \quad\left(\subset\left\{u_{j k}\right\}\right)$ is convergent in $L^{q}\left(\boldsymbol{\Omega}_{k}\right) \quad\left(1 \leq q<p^{*}\right)$, and $|f(x, t)|^{n p /(n+p)} \leq C|t|^{\alpha n p /(n+p)}$ with $1<\alpha n p /(n+p)<p^{*}$, whence for $j, k$ sufficiently large, $\left\|f\left(\cdot, u_{j j}\right)-f\left(\cdot, u_{i i}\right)\right\|_{L^{n p /(n+p)}\left(\Omega_{k}\right)}<\varepsilon$. The compactness of $K^{\prime}$ follows immediately from (6) by replacing $u_{j}$ with $u_{j j}$ and $u$ with $u_{i i}$.

The critical points $u$ of $J$, i.e.,

$$
J^{\prime}(u)(\varphi)=\int_{\Omega}\left(a|\nabla u|^{p-2} \nabla u \cdot \nabla \varphi+b|u|^{p-2} u \varphi-f(x, u) \varphi\right) d x=0
$$

for all $\varphi \in E$ are weak solutions of $l u=f(x, u)$.

Lemma 2. Let $u$ be a critical point of $J$.

(a) $u \in L^{q}(\Omega), \frac{n p}{n-p} \leq q<\infty$. If $b(x) \geq b_{0}>0$, then $u \in L^{q}(\Omega)$, $p \leq q<\infty$,

(b) $\lim _{|x| \rightarrow \infty} u=0$.

Proof. (a) Let $u^{+}(x)=\max \{u(x), 0\}, u^{-}(x)=\max \{-u(x), 0\}$. We show that statement $(\mathrm{a})$ is true for $u^{ \pm}$. Set $u_{k}(x)=\min \left\{u^{ \pm}(x), k\right\}, k=1,2, \cdots$. For any real $i \geq 1,\left(u_{k}\right)^{i} \in E$. Substituting $\varphi=\left(u_{k}\right)^{i}$ in (7) and using $-\|g\|_{\infty}|t|^{\alpha} \leq f(x, t) \leq\|g\|_{\infty}|t|^{\alpha}$, we obtain

$$
i \int_{\Omega}\left(u_{k}\right)^{i-1}\left|\nabla u_{k}\right|^{p} \leq \frac{\|g\|_{\infty}}{a_{0}} \int_{\Omega}\left(u^{ \pm}\right)^{\alpha+i} .
$$

By the fact that $\left(u_{k}\right)^{i-1}\left|\nabla u_{k}\right|^{p}=\left(\frac{p}{i+p-1}\right)^{p}\left|\nabla\left(u_{k}\right)^{(i+p-1) / p}\right|^{p}$ and Sobolev's Inequality, we have

$$
\left(\int_{\Omega}\left(u_{k}\right)^{\frac{n}{n-p}(i+p-1)}\right)^{\frac{n-p}{n}} \leq C \int_{\Omega}\left(u^{ \pm}\right)^{\alpha+i},
$$

where $C=C\left(n, i, p, \alpha, a_{0},\|g\|_{\infty}\right)$. Setting

$$
\begin{gathered}
\sigma=p^{*}-1-\alpha, \quad i=i_{0}=1+\sigma, \\
q_{0}=\frac{n}{n-p}\left(i_{0}+p-1\right)=\frac{n}{n-p}(p+\sigma),
\end{gathered}
$$

and letting $k \rightarrow \infty$ in $(8)$, we conclude that $u^{ \pm} \in L^{q_{0}}(\Omega)$. Iterating this process gives

$$
\left(\left\|u_{k}\right\|_{q_{j+1}}\right)^{\frac{n-p}{n}} \leq C \int_{\Omega}\left(u^{ \pm}\right)^{q_{j}}
$$


where $q_{j}=\frac{n}{n-p}\left(i_{j}+p-1\right), i_{j}=1+\sigma+\frac{n}{n-2} \sigma+\cdots+\left(\frac{n}{n-2}\right)^{j} \sigma$, and where $C=$ $C\left(n, i_{j}, p, \alpha, a_{0},\|g\|_{\infty}\right), j=0,1, \cdots$. Thus, $u^{ \pm} \in L^{q}(\Omega), \frac{n p}{n-p} \leq q<\infty$, and (a) follows. To show (b), we choose $r_{0}$ sufficiently large so that $B_{2}(x) \subseteq \Omega$ for all $x$ such that $|x| \geq r_{0}$. Then by Theorem 1 of Serrin [13], for some $q>\frac{n}{p}$,

$$
\|u\|_{L^{\infty}\left(B_{1}(x)\right)} \leq C\left\{\|u\|_{L^{p^{*}\left(B_{2}(x)\right)}}+\|f(x, u)\|_{L^{q}\left(B_{2}(x)\right)}\right\}
$$

where $C=C(n, p, q)$. The decay of $u$ follows.

Now we can employ the Mountain Pass Theorem to obtain a solution of $(*)$.

Theorem 1. Under conditions (i)-(iii), the problem (*) has a positive decaying solution $u \in C^{1, \delta}\left(\bar{\Omega} \cap B_{r}(0)\right)$ for any $r>0$ and some $\delta=\delta(r) \in(0,1)$.

Proof. Since we seek positive solutions, it is convenient to define $f(x, t)=0$ for $t \leq 0$. By condition (ii), for small $r>0$, there exists a $c>0$ such that

$$
J(u) \geq \frac{1}{p}\|u\|_{l}^{p}-C\|g\|_{p_{0}}\|u\|_{l}^{\alpha+1} \geq c
$$

for $u \in \partial B_{r}(0)$. Integrating and using conditions (i) and (iii), we see that $F(x, t) \geq a_{1} t^{\mu}-a_{2}$ for $(x, t) \in \Omega_{0} \times R^{+}$, some $a_{1}, a_{2}>0$. Let $\varphi \in C_{0}^{\infty}\left(\Omega_{0}\right)$ such that $\varphi(x) \geq 0, \not \equiv 0$, and let $s \in R^{+}$. For $s$ large,

$$
J(s \varphi) \leq \frac{1}{p} s^{p}\|\varphi\|_{l}^{p}-s^{\mu} \int_{\Omega_{0}} a_{1} \varphi^{\mu}+a_{2}\left|\Omega_{0}\right|<0 .
$$

Thus we obtain the existence of $e$ with $J(e)<0$. To see that the (PS) condition holds, suppose $\left\{u_{i}\right\} \subseteq E$ is such that $J\left(u_{i}\right) \leq C$ and $J^{\prime}\left(u_{i}\right)(\cdot) \rightarrow 0$. Note that the inequality

$$
C \geq J\left(u_{i}\right) \geq \frac{1}{p}\left\|u_{i}\right\|_{l}^{p}-\frac{1}{\mu} \int_{\Omega} f\left(x, u_{i}\right) u_{i} \geq\left(\frac{1}{p}-\frac{1}{\mu}\right)\left\|u_{i}\right\|_{l}^{p}+\frac{1}{\mu} J^{\prime}\left(u_{i}\right)\left(u_{i}\right)
$$

yields the boundedness of $\left\{u_{i}\right\}$. Consequently, it follows from the compactness of $K^{\prime}$ that there exists a subsequence of $\left\{u_{i}\right\}$, say $\left\{u_{i}\right\}$ itself, such that $K^{\prime}\left(u_{i}\right)$ is Cauchy in $E^{*}$. We claim that $\left\{u_{i}\right\}$ is a Cauchy sequence in $E$. Indeed, we have the inequality

$$
|\xi-\eta|^{p} \leq \begin{cases}\left(|\xi|^{p-2} \xi-|\eta|^{p-2} \eta\right) \cdot(\xi-\eta) & \text { if } p \geq 2, \\ {\left[\left(|\xi|^{p-2} \xi-|\eta|^{p-2} \eta\right) \cdot(\xi-\eta)\right]^{\frac{p}{2}}\left[|\xi|^{p}+|\eta|^{p}\right]^{\frac{2-p}{2}}} & \text { if } 1<p<2\end{cases}
$$

for $\xi, \eta \in R^{n}$ (see, e.g., [7, 14]). On the other hand, from (7) we obtain

$$
\begin{aligned}
\int_{\Omega} a\left(\left|\nabla u_{i}\right|^{p-2} \nabla u_{i}-\left|\nabla u_{j}\right|^{p-2} \nabla u_{j}\right) \cdot\left(\nabla u_{i}-\nabla u_{j}\right) \\
\quad+b\left(\left|u_{i}\right|^{p-2} u_{i}-\left|u_{j}\right|^{p-2} u_{j}\right) \cdot\left(u_{i}-u_{j}\right) \\
\leq\left|J^{\prime}\left(u_{i}\right)\left(u_{i}-u_{j}\right)\right|+\left|J^{\prime}\left(u_{j}\right)\left(u_{i}-u_{j}\right)\right|+\left|\int_{\Omega}\left(f\left(x, u_{i}\right)-f\left(x, u_{j}\right)\right)\left(u_{i}-u_{j}\right)\right| \\
\leq C\left\{\left\|J^{\prime}\left(u_{i}\right)\right\|_{E^{*}}+\left\|J^{\prime}\left(u_{j}\right)\right\|_{E^{*}}+\left\|K^{\prime}\left(u_{i}\right)-K^{\prime}\left(u_{j}\right)\right\|_{E^{*}}\right\},
\end{aligned}
$$

where $C=C(n, p)$. From (9) and (10), it follows immediately that $\left\{u_{i}\right\}$ is Cauchy in $E$. Thus the (PS) condition holds. The Mountain Pass Theorem guarantees the existence of a nontrivial critical point of $J$, say $u$. By Lemma $2, u$ decays. Letting $\varphi=u^{-}$in (7) implies that $u \geq 0$ in $\Omega$. The positivity $u(x)>0$ in $\Omega$ follows from the Harnack type inequality [15, Theorem 1.1]. Finally, the proof of [9, Theorem 1] implies that $u \in C^{1, \delta}\left(\bar{\Omega} \cap B_{r}(0)\right), r>0$. 
To see this, let $r$ be so large that $\partial \Omega \subset B_{r}(0)$. Since $\left.u\right|_{\partial \Omega}=0$, the boundary regularity arguments of [9, Theorem 1] can be applied in a neighborhood of $\partial \Omega$ in $\bar{\Omega} \cap B_{r}(0)$, while the interior regularity arguments of $[9$, Theorem 1] can be applied in the rest part of $\bar{\Omega} \cap B_{r}(0)$.

\section{OTHER PROBLEMS}

We employ the method of the previous section, with some modifications, to consider the problem $(*)$ in cases $(2)$ and $(3)$

$$
f(x, u)= \begin{cases}h(x) u^{\beta}, & 0 \leq \beta<p-1, \\ g(x) u^{\alpha}+h(x) u^{\beta}, & 0 \leq \beta<p-1<\alpha<p^{*}-1 .\end{cases}
$$

We introduce the following conditions:

(iv) $0 \leq f(x, t) \leq h(x)|t|^{\beta}, 0 \leq \beta<p-1, h \in L^{\infty}(\Omega) \cap L^{q_{0}}(\Omega), q_{0}=$ $\frac{n p}{n p-(\beta+1)(n-p)}$

(v) $f(x, t) \geq h_{0}(x) t^{\beta_{0}}$ as $t \rightarrow 0^{+}, 0 \leq \beta_{0} \leq \beta, h_{0}(x) \geq 0, \not \equiv 0$.

Theorem 2. Under conditions (i), (iv), and (iv), problem (*) has a positive decaying solution $u \in C^{1, \delta}\left(\bar{\Omega} \cap B_{r}(0)\right)$ for any $r>0$ and some $\delta=\delta(r) \in(0,1)$. Proof. We assume $f(x, t)=f(x, 0)$ for $t \leq 0$ and define the functionals $K(u)$ and $J(u)$ on $E$ as before. By condition (iv), the functional $J$ is weakly lower semicontinuous differentiable. Moreover, $J$ is bounded below, since

$$
J(u) \geq \frac{1}{p}\|u\|_{l}^{p}-C\|h\|_{q_{0}}\|u\|_{l}^{\beta+1} .
$$

Thus $J$ has a critical point $u: J(u)=\inf \{J(v) \mid v \in E\}$, which is a solution of $(*)$. We note that $u$ must be nontrivial since

$$
J(s \varphi) \leq \frac{s^{p}}{p}\|\varphi\|_{l}^{p}-\frac{s^{\beta_{0}+1}}{\beta_{0}+1} \int_{\Omega} h_{0}(x)|\varphi|^{\beta_{0}+1}<0
$$

for some $\varphi \in C_{0}^{\infty}(\Omega)$ and small $s>0$. The arguments of the nonnegativity, regularity, and decay of $u$ in Theorem 1 work here. Since $l u \geq 0$, the weak Harnack inequality [15, Theorem 1.2] yields $u(x)>0$ in $\Omega$.

Theorem 3. Let $f(x, t)=f_{1}(x, t)+f_{2}(x, t)$. Suppose that $f_{1}$ satisfies (i)-(iii) and $f_{2}$ satisfies (i), (iv), and (v). Then the problem (*) has two positive decaying solutions $u_{1}, u_{2} \in C^{1, \delta}\left(\bar{\Omega} \cap B_{r}(0)\right)$ for any $r>0$ and some $\delta=\delta(r) \in(0,1)$ provided

$$
\begin{aligned}
2 A^{p}\|g\|_{p_{0}}^{\frac{p-\beta-1}{\alpha-\beta}} \cdot\|h\|_{q_{0}}^{\frac{\alpha+1-p}{\alpha-\beta}} & {\left[\frac{1}{\alpha+1}\left(\frac{(\alpha+1)(p-\beta+1)}{(\beta+1)(\alpha+1-p)}\right)^{\frac{\alpha+1-p}{\alpha-\beta}}\right.} \\
& \left.+\frac{1}{\beta+1}\left(\frac{(\beta+1)(\alpha+1-p)}{(\alpha+1)(p-\beta-1)}\right)^{\frac{p-\beta-1}{\alpha-\beta}}\right]<1,
\end{aligned}
$$

where $A=\frac{n-1}{n v_{n}^{1 / n}} \frac{\Gamma(n / p-1)}{\Gamma(n / p)}, v_{n}=\operatorname{vol}\left(B_{1}(0)\right)$.

Proof. Once again, we employ Mountain Pass arguments to obtain the first nontrivial critical point of $J$. Here we assume $f(x, t)=f(x, 0)$ for $t \leq 0$. In this case,

$$
J(u)=\frac{1}{p}\|u\|_{l}^{p}-\int_{\Omega} K(u), \quad K(u)=\int_{\Omega} F_{1}(x, u)+\int_{\Omega} F_{2}(x, u),
$$


where $F_{1}(x, u)=\int_{0}^{u} f_{1}(x, s) d s, F_{2}(x, u)=\int_{0}^{u} f_{2}(x, s) d s . J$ is weakly lower semicontinuous and differentiable, while $K^{\prime}$ is compact. Observe that for some $(0 \leq) \varphi \in C_{0}^{\infty}\left(\Omega_{0}\right)$,

$$
J(s \varphi) \leq \frac{1}{p} s^{p}\|\varphi\|_{l}^{2}-s^{\mu} \int_{\Omega} a_{1} \varphi^{u}+a_{2}\left|\Omega_{0}\right|<0
$$

for large $s>0$ and that

$$
\begin{aligned}
J(u)= & \frac{1}{p}\|u\|_{l}^{p}-\int_{\Omega} F_{1}(x, u)-\int_{\Omega} F_{2}(x, u) \\
= & \left(\frac{1}{p}-\frac{1}{\mu}\right)\|u\|_{l}^{p}+\frac{1}{\mu} J^{\prime}(u)(u)+\int_{\Omega}\left(\frac{1}{\mu} f_{1}(x, u) u-F_{1}(x, u)\right) \\
& +\int_{\Omega}\left(\frac{1}{\mu} f_{2}(x, u) u-F_{2}(x, u)\right) \\
\geq & \left(\frac{1}{p}-\frac{1}{\mu}\right)\|u\|_{l}^{p}+\frac{1}{\mu} J^{\prime}(u)(u)-C\left(1+\frac{1}{\mu}\right)\|h\|_{q_{0}}\|u\|_{l}^{\beta+1} .
\end{aligned}
$$

It follows that any sequence $\left\{u_{i}\right\}$ such that $J\left(u_{i}\right) \leq C$ and $J^{\prime}\left(u_{i}\right) \rightarrow 0$ is bounded. Thus $\left\{u_{i}\right\}$ has a convergent subsequence by the compactness of $K^{\prime}$ and $J^{\prime}\left(u_{i}\right) \rightarrow 0$. The (PS) condition now follows, but the step $J(u) \geq a$ for $u \in \partial B_{r}(0)$ no longer follows as before. However,

$$
\begin{aligned}
\left.J(u)\right|_{\|u\|_{l}=r} & \geq\left.\left(\frac{1}{p}\|u\|_{l}^{p}-\frac{1}{\alpha+1} A^{\alpha+1}\|g\|_{p_{0}}\|u\|_{l}^{\alpha+1}-\frac{1}{\beta+1} A^{\beta+1}\|h\|_{q_{0}}\|u\|_{l}^{\beta+1}\right)\right|_{\|u\|_{l}=r} \\
& =\frac{1}{p} r^{p}\left(1-\frac{p}{\alpha+1} A^{\alpha+1}\|g\|_{p_{0}} r^{\alpha+1-p}-\frac{p}{\beta+1} A^{\beta+1}\|h\|_{q_{0}} r^{\beta+1-p}\right) \\
& \equiv \frac{1}{p} r^{p} H(r) .
\end{aligned}
$$

Elementary differentiation shows that $H(r)$ has an absolute maximum

$$
r_{0}=\frac{1}{A}\left[\frac{\left(\alpha+1(p-1-\beta)\|h\|_{q_{0}}\right.}{(\beta+1)(\alpha+1-p)\|g\|_{p_{0}}}\right]^{\frac{1}{\alpha-\beta}}
$$

By assumption, $H\left(r_{0}\right)>0$. Hence $\left.J(u)\right|_{\|u\|_{l}=r_{0}}>0$. By the Mountain Pass Theorem, $J(\cdot)$ has a critical point $u_{1}$ with $J\left(u_{1}\right)>0$. Observe $\left.J(u)\right|_{\|u\|_{l}=r_{0}}>0$ and

$$
J(s \varphi) \leq \frac{s^{p}}{p}\|\varphi\|_{l}^{p}+s^{\alpha+1} C\|g\|_{p_{0}}\|\varphi\|_{l}^{\alpha+1}-\frac{s^{\beta_{0}+1}}{\beta_{0}+1} \int_{\Omega} h_{0}(x)|\varphi|^{\beta_{0}+1}<0
$$

for some $\varphi \in C_{0}^{\infty}(\Omega)$ and small $s>0$. It follows that $J(\cdot)$ attains its local minimum at some $u_{2} \in B_{r_{0}}(0)$, i.e., $J\left(u_{2}\right)=\inf \left\{J(v) \mid v \in B_{r_{0}}(0)\right\}<0$. As before, we have $u_{1}, u_{2} \geq 0$. Since $u_{1}, u_{2}$ satisfy $l u \geq f_{1}(x, u)$, the positivity of $u_{1}, u_{2}$ follows from [15, Theorem 1.2]. A slightly modified proof of Lemma 2 shows the decay of $u_{1}, u_{2}$; in this case the estimate of the proof of Lemma 
2(a) proceeds as follows: $i \geq 1$,

$$
\begin{aligned}
\int_{\Omega} f(x, u) u^{i} & =\int_{\Omega} f_{1}(x, u) u^{i}+\int_{\Omega} f_{2}(x, u) u^{i} \\
& \leq\|g\|_{\infty} \int_{\Omega} u^{\alpha+i}+\int_{0 \leq u \leq 1} h u^{\beta+i}+\int_{1 \leq u} h u^{\beta+i} \\
& \leq\left(\|g\|_{\infty}+\|h\|_{\infty}\right) \int_{\Omega} u^{\alpha+i}+\int_{\Omega} h u^{\beta+1} \\
& \leq\left(\|g\|_{\infty}+\|h\|_{\infty}\right) \int_{\Omega} u^{\alpha+i}+C\|h\|_{q_{0}}\|u\|_{l}^{\beta+1}
\end{aligned}
$$

The rest is the same. This completes the proof.

Suppose that $g(x)=O\left(|x|^{-\nu}\right), h(x)=O\left(|x|^{-\gamma}\right)$ at $\infty$. Then conditions (ii) and (iv) imply that

$$
\nu>\frac{n p-(\alpha+1)(n-p)}{p}, \quad \gamma>\frac{n p-(\beta+1)(n-p)}{p} .
$$

If $b(x) \geq b_{0}>0$, the assumption $g \in L^{p_{0}}(\Omega)$ of (ii) could be replaced by $\|g\|_{L^{1}\left(B_{1}(x)\right)} \rightarrow 0$ as $|x| \rightarrow \infty$. In this case, we need only apply the embedding theorem of [3, Theorem 2.3] in the proofs above, where the property that $\|g\|_{L^{p_{0}\left(\Omega \backslash \Omega_{k}\right)}}$ can be arbitrarily small is used.

\section{ACKNOWLEDGMENTS}

The author wishes to thank his supervisor, Professor W. Allegretto, for guidance and encouragement and the referee for valuable suggestions.

\section{REFERENCES}

1. R. A. Adams, Sobolev spaces, Academic Press, New York, 1975.

2. J. P. G. Azorero and I. P. Alonso, Existence and nonexistence for the p-Laplacian nonlinear eigenvalues, Comm. Partial Differential Equations 12 (1987), 1389-1430.

3. M. Berger and M. Schechter, Embedding theorems and quasi-linear elliptic boundary value problems for unbounded domains, Trans. Amer. Math. Soc. 172 (1972), 261-278.

4. M.-F. Bidaut-Veron, Local and global behavior of solutions of quasilinear equations of Emden-Fowler type, Arch. Rational Mech. Anal. 107 (1989), 293-324.

5. $\mathrm{H}$. Egnell, Existence and nonexistence results for $m$-Laplace equations involving critical Sobolev exponents, Arch. Rational Mech. Anal. 104 (1988), 57-77.

6. M. Guedda and L. Veron, Quasilinear elliptic equation involving critical Sobolev exponents, Nonlinear Anal. T.M.A. 13 (1989), 879-902.

7. S. Kichenassamy and L. Veron, Singular solutions of the p-Laplace equation, Math. Ann. 275 (1985), 599-615.

8. G. Li and $\mathrm{S}$. Yan, Eigenvalue problems for quasilinear elliptic equations on $R^{N}$, Comm. Partial Differential Equations 14 (1989), 1291-1314.

9. G. M. Lieberman, Boundary regularity for solutions of degenerate elliptic equations, Nonlinear Anal. T. M. A. 12 (1988), 1203-1219.

10. V. G. Maz'ja, Sobolev spaces, Springer-Verlag, Berlin, Heidelberg, New York, and Tokyo, 1985.

11. W.-M . Ni and J. Serrin, Existence and nonexistence theorems for ground states of quasilinear partial differential equations. The anomalous case, Accad. Naz. Lincei 77 (1986), 231-257.

12. P. H. Rabinowitz, Minimax methods in critical point theory with applications to differential equations, Amer. Math. Soc., Providence, RI, 1986. 
13. J. Serrin, Local behavior of solutions of quasilinear equations, Acta Math. 111 (1964), 247302.

14. F. De. Thelin, Local regularity properties for the solutions of a nonlinear partial differential equation, Nonlinear Anal. T. M. A. 6 (1982), 839-844.

15. N. S. Trudinger, On harnack type inequalities and their applications to quasilinear elliptic equations, Comm. Pure Appl. Math. 20 (1967), 721-747.

Department of Mathematics, University of Alberta, Edmonton, Alberta, Canada T6G 2G1

Current address, Lao Sen Yu: Department of Mathematics, The University of British Columbia, Vancouver, British Columbia, Canada V6T 1Y4

E-mail address: userlsyu@mtsg.ubc.ca 